\title{
Dialysis attendance patterns and health care utilisation of Aboriginal patients attending dialysis services in urban, rural and remote locations
}

Gillian Gorham ${ }^{1 *}$, Kirsten Howard ${ }^{2,3}$, Joan Cunningham¹, Paul Damian Lawton ${ }^{1}$, A. M. Shamsir Ahmed ${ }^{4}$, Federica Barzi ${ }^{5}$ and Alan Cass ${ }^{1}$

\begin{abstract}
Background: Aboriginal people in the Northern Territory (NT) suffer the heaviest burden of kidney failure in Australia with most living in remote areas at time of dialysis commencement. As there are few dialysis services in remote areas, many Aboriginal people are required to relocate often permanently, to access treatment. Missing dialysis treatments is not uncommon amongst Aboriginal patients but the relationship between location of dialysis service and dialysis attendance (and subsequent hospital use) has not been explored to date.
\end{abstract}

Aim: To examine the relationships between location of dialysis service, dialysis attendance patterns and downstream health service use (overnight hospital admissions, emergency department presentations) among Aboriginal patients in the NT.

Methods: Using linked hospital and dialysis registry datasets we analysed health service activity for 896 Aboriginal maintenance dialysis patients in the NT between 2008 and 2014. Multivariate linear regression and negative binomial regression analyses explored the associations between dialysis location, dialysis attendance and health service use.

Results: We found missing two or more dialysis treatments per month was more likely for Aboriginal people attending urban services and this was associated with a two-fold increase in the rate of hospital admissions and more than three-fold increase in ED presentations. However, we found higher dialysis attendance and lower health service utilisation for those receiving care in rural and remote settings. When adjusted for age, time on dialysis, region, comorbidities and residence pre-treatment, among Aboriginal people from remote areas, those dialysing in remote areas had lower rates of hospitalisations (IRR 0.56; $P<0.001)$ when compared to those who relocated and dialysed in urban areas.

Conclusion: There is a clear relationship between the provision and uptake of dialysis services in urban, rural and remote areas in the NT and subsequent broader health service utilisation. Our study suggests that the low dialysis attendance associated with relocation and care in urban models for Aboriginal people can potentially be ameliorated by access to rural and remote models and this warrants a rethinking of service delivery policy. If providers are to

\footnotetext{
*Correspondence: gillian.gorham@menzies.edu.au

${ }^{1}$ Menzies School of Health Research, Charles Darwin University,

Darwin 0810, Australia
}

Full list of author information is available at the end of the article

(C) The Author(s) 2022. Open Access This article is licensed under a Creative Commons Attribution 4.0 International License, which permits use, sharing, adaptation, distribution and reproduction in any medium or format, as long as you give appropriate credit to the original author(s) and the source, provide a link to the Creative Commons licence, and indicate if changes were made. The images or other third party material in this article are included in the article's Creative Commons licence, unless indicated otherwise in a credit line to the material. If material is not included in the article's Creative Commons licence and your intended use is not permitted by statutory regulation or exceeds the permitted use, you will need to obtain permission directly from the copyright holder. To view a copy of this licence, visit http://creativecommons.org/licenses/by/4.0/. The Creative Commons Public Domain Dedication waiver (http://creativeco mmons.org/publicdomain/zero/1.0/) applies to the data made available in this article, unless otherwise stated in a credit line to the data. 


\section{Introduction}

The burden of chronic kidney disease disproportionately affects socioeconomically disadvantaged populations [1-3]. In Australia, the Northern Territory (NT) has the highest rates of kidney failure, with Aboriginal people who live in remote areas requiring kidney treatment at more than 10 times national rates [4].

The uptake of home therapies (self-care haemodialysis and peritoneal dialysis) is significantly lower in the NT compared to other Australian jurisdictions and few Aboriginal people receive a transplant. Thus most Aboriginal patients receive dialysis in a staffed facility [5]. Until recently there were few staffed dialysis services available in rural and remote areas [6] where the burden of kidney disease is heaviest. With renal and dialysis services centralised in the urban areas in the NT, most Aboriginal people with kidney failure must move, often permanently, for treatment.

Existing research indicates that a model of dialysis care that requires a person to permanently relocate away from their usual residence and support networks has significant negative consequences for the individual and their community [7-12]. Family and cultural responsibilities, coupled with distance and cost of remote travel often manifest in missed dialysis treatments in urban areas [13, 14]. Consistently lower rates of dialysis attendance are thought to be associated with higher hospitalisations [15, 16] but there has been little examination of the difference in dialysis attendance rates by location or how this may impact on other health service utilisation.

\section{Objective}

We aimed to examine the relationship between the type and location of a dialysis model of care and broader health service utilisation, defined as dialysis treatments, hospital admissions, days in hospital and emergency department presentations. Of particular interest were patterns of health service utilisation for patients from remote areas who needed to relocate to receive care in urban locations, compared to patients who were able to access dialysis services closer to home.

\section{Methods}

The NT is a large land mass of over 1.3million square kilometres with a small and sparsely dispersed population of fewer than 250,000 people. Most of the NT is classified as remote and very remote [17]. Most people live in the two main urban centres of Darwin and Alice Springs; but the majority (70\%) of Aboriginal people, who make up approximately $30 \%$ of the population, live in remote and very remote communities.

Renal services are configured on a hub and spoke model with a hub based in the tertiary hospitals in Darwin and Alice Springs, $2000 \mathrm{~km}$ apart. The hubs oversee care delivered in urban, rural and remote satellite centres, notionally described as spokes. At the time of this study, most staffed dialysis services were centralised in the urban areas with only limited dialysis services available outside of these areas despite the significant demand. Renal patients relocate from more than 50 remote communities, including across jurisdictional boundaries, in order to access services (Fig. 1).

For the purposes of this study and ease of differentiation between services, we categorised dialysis services by location (urban, rural and remote) and support type and allocated services to a model of care $(\mathrm{DxMoC})$. Table 1 describes the characteristics of the DxMoC depicted in Fig. 1. Transplantation is not a dialysis model of care and was not included in the study.

\section{Overall study design}

We conducted a retrospective analysis of hospital and registry data to examine health service utilisation (dialysis attendance rates, hospital admissions, days in hospital and emergency department presentations) of maintenance dialysis patients in the NT between 2008 to 2014 . The study received Ethics approval from the Joint Department of Health and Menzies School of Health Research Human Research Ethics Committee (HREC 2015-2334) and the Central Australian Health Research Ethics Committee (HREC 15-283).

\section{Data sources and linkage}

We used two linked longitudinal data sets: the NT Department of Health's (DoH) Admitted Patient Care (APC) (hospital) dataset which contains individual episodes of patient care for the five (public) hospitals and all satellite services in the NT; and the Australia and New Zealand Dialysis and Transplant Registry (ANZDATA) dataset which contains patient level data for people receiving maintenance kidney replacement therapy (KRT) in Australia and New Zealand. The ANZDATA dataset is based on a voluntary annual census from participating renal units.

The combined full database population (linked as part of the broader study [18]) included: 1) any individual 


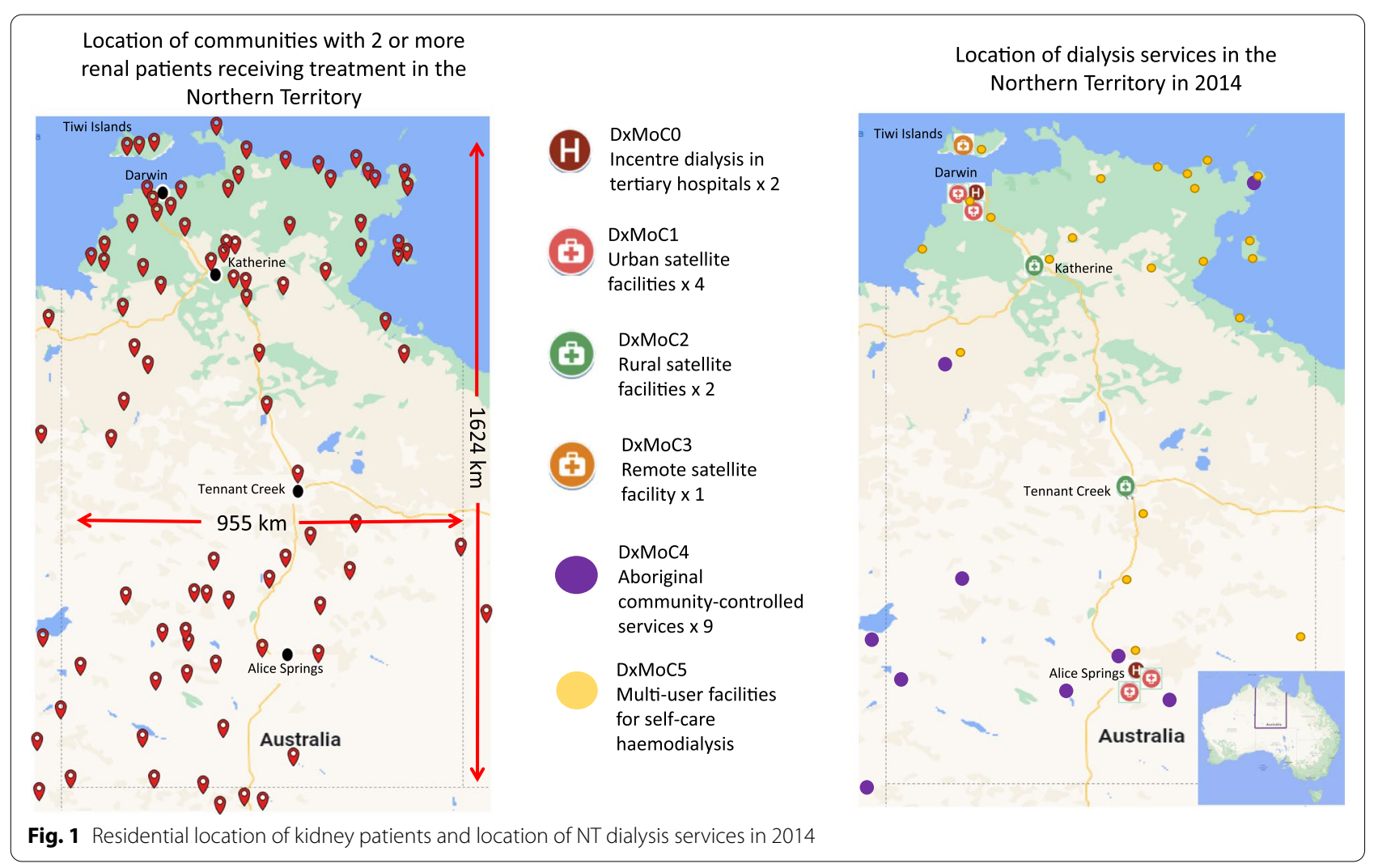

from the APC dataset with a diagnosis or procedure code for dialysis or transplantation (ESM Table S1) based on the International Classification of Diseases version 10, Australian Modification (ICD 10 AM) between the years 2000 and $2015(n=2844)$; and 2$)$ any individual from the ANZDATA dataset who registered as ever having KRT in the NT between 2000 and $2015(n=1390)$.

Linkage of two additional activity data sets was necessary: a) interstate patient travel information $(n=171)$; and b) dialysis data from individuals $(n=189)$ receiving self-care haemodialysis (DxMoC5) and/or care in the remote community-controlled service (DxMoC4), as this information was known to be inconsistently captured in the hospital data set.

\section{Study cohort definition}

This study included Aboriginal adults (over the age of 16 years as of 1st Jan 2008), receiving maintenance dialysis (defined as chronic haemodialysis or peritoneal dialysis continuously for greater than 3 months), between 2008 and 2014 inclusive. This date range was chosen as some models of care only became fully established after 2008 and the additional activity data (for DxMoC4 and DxMoC5) was only available to the end of 2014.

Non-Aboriginal dialysis patients were excluded as they comprised less than $10 \%$ of the dialysis population and were not represented in three (rural and remote models) of the seven models of care. Patients were also excluded if: they received acute dialysis treatments only; were interstate visitors; or did not have at least one admission after 2008, to exclude patients who left the NT permanently. Patients were censored if they had an active transplant (Fig. 2).

Additional information regarding setting, data sources, linkage and methods can be found in the Electronic Supplementary Material (ESM).

\section{Variable definitions}

Besides the exposure variables of gender, age at time of admission, time on dialysis at admission, we were also interested in region (Top End or Central Australia to align with health service responsibility); residence preKRT (defined as the earliest admission address in the 24 months pre-KRT start); and relocation (defined as an indefinite change in residence from remote area pre-KRT to an urban address post commencement of maintenance dialysis). Our process may have underestimated 'relocation' as many remote residing people will move closer to specialist care as their health needs increase but before they require KRT [19]. Residences were mapped to the Modified Monash Model (MMM) which categorises 
Table 1 Dialysis services in the NT characterised as Dialysis Models of Care (DxMoC)

\begin{tabular}{|c|c|c|}
\hline Dialysis Model & Description & Characteristics \\
\hline $\begin{array}{l}\text { DxMoCO } \\
\text { In-centre dialysis }\end{array}$ & $\begin{array}{l}\text { Hub service: situated in tertiary } \\
\text { centre: acute and maintenance } \\
\text { haemodialysis ( } 8-12 \text { stations) }\end{array}$ & $\begin{array}{l}\text { Majority of patients commence } \\
\text { treatment here: used for complex } \\
\text { patients and overflow from satellite } \\
\text { centres. (S/P* }=1: 2 \text { or } 1: 3)\end{array}$ \\
\hline $\begin{array}{l}\text { DxMoC1 } \\
\text { Urban Satellite Unit }\end{array}$ & $\begin{array}{l}\text { Large facilities in urban areas: } \\
\text { maintenance haemodialysis (22-26 } \\
\text { stations) }\end{array}$ & $\begin{array}{l}\text { Provides majority of services, default } \\
\text { service when rural and remote services } \\
\text { at capacity }\left(S / P^{*}=1: 4\right)\end{array}$ \\
\hline $\begin{array}{l}\text { DxMoC2 } \\
\text { Rural Satellite Unit }\end{array}$ & $\begin{array}{l}\text { Smaller facilities: often co-located } \\
\text { with regional hospitals: maintenance } \\
\text { haemodialysis ( } 8-16 \text { stations) }\end{array}$ & $\begin{array}{l}\text { Usually for stable patients: generally a } \\
\text { waiting list }\left(S / P^{*}=1: 3 \text { or } 1: 4\right)\end{array}$ \\
\hline $\begin{array}{l}\text { DxMoC3 } \\
\text { Remote Satellite Unit }\end{array}$ & $\begin{array}{l}\text { Small units isolated from hub by } \\
\text { distance or geography: maintenance } \\
\text { haemodialysis ( } 6-8 \text { stations) }\end{array}$ & $\begin{array}{l}\text { Usually reserved for clinically well, } \\
\text { physically mobile patients: generally a } \\
\text { waiting list }\left(S / P^{*}=1: 3\right)\end{array}$ \\
\hline $\begin{array}{l}\text { DxMoC4 } \\
\text { Remote CC* Satellite } \\
\text { Unit }\end{array}$ & $\begin{array}{l}\text { Aboriginal owned and determined; } \\
\text { small remote based units: permanent } \\
\text { and respite dialysis with social } \\
\text { supports (2-4 stations) }\end{array}$ & $\begin{array}{l}\text { Patient acceptance criteria less } \\
\text { restrictive as more support services } \\
\text { available: generally a waiting list } \\
\left(\mathrm{S} / \mathrm{P}^{*}=1: 2\right)\end{array}$ \\
\hline $\begin{array}{l}\text { DxMoC5 } \\
\text { Self-care HD* }\end{array}$ & $\begin{array}{l}\text { Multi-user } 2 \text { station facilities } \\
\text { (unstaffed) in remote areas provide } \\
\text { safe/secure dialysis infrastructure for } \\
\text { self-care patients }\end{array}$ & $\begin{array}{l}\text { Infrastructure managed from hub. } \\
\text { Training and regular onsite visits } \\
\text { provided by hub service for self-care } \\
\text { patients. (S/P* = NA) }\end{array}$ \\
\hline $\begin{array}{l}\text { DxMoC6 } \\
\text { Self-care PD* }\end{array}$ & $\begin{array}{l}\text { Independent or carer managed } \\
\text { peritoneal dialysis in own home with } \\
\text { regular onsite visits from hub staff. }\end{array}$ & $\begin{array}{l}\text { Training and ongoing support provided } \\
\text { by hub service for peritoneal dialysis } \\
\text { patients. }\left(\mathrm{S} / \mathrm{P}^{*}=\mathrm{NA}\right)\end{array}$ \\
\hline
\end{tabular}

*CC: Community controlled; HD: haemodialysis; PD: peritoneal dialysis; S/P: Staff to Patient Ratio 


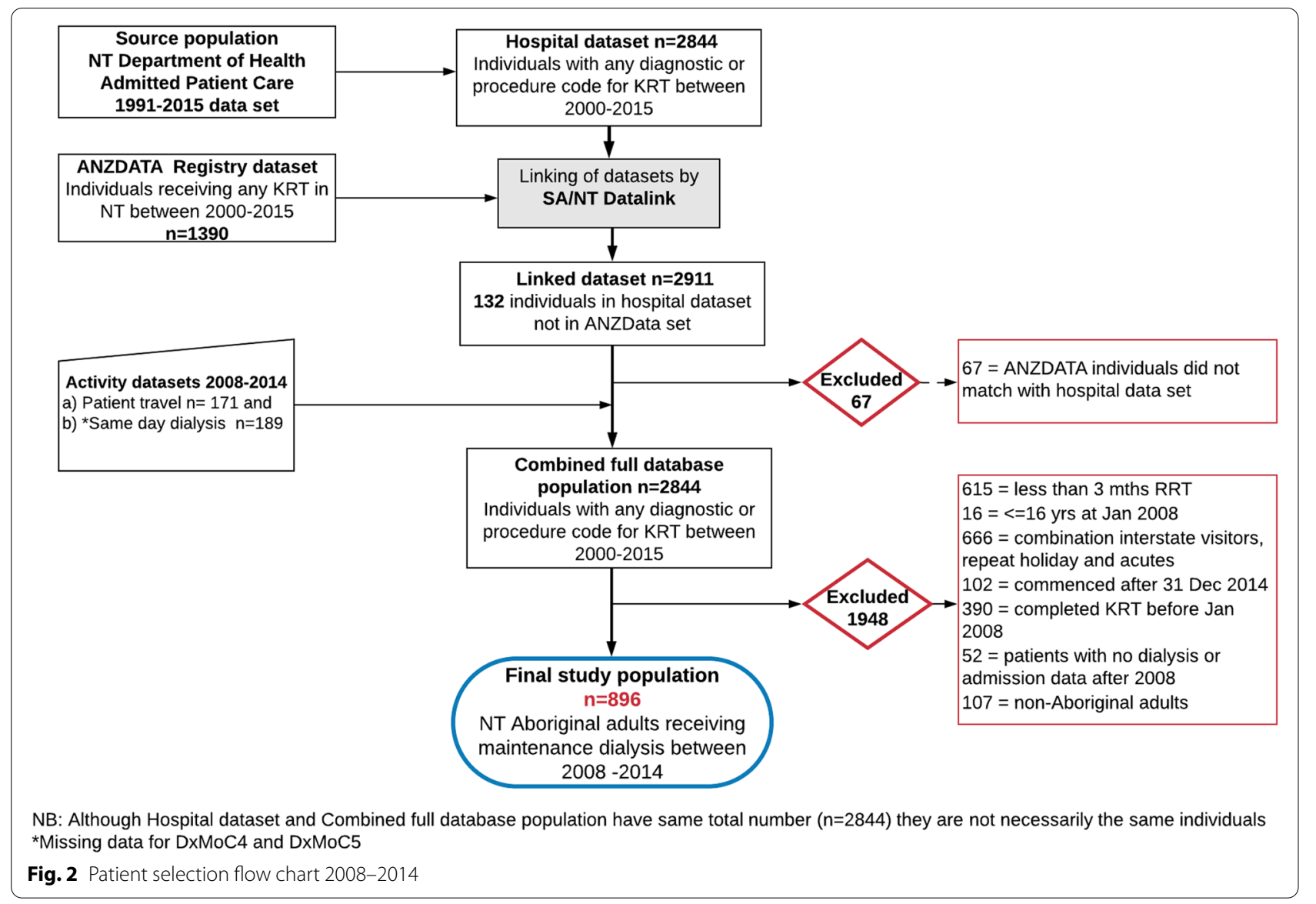

areas according to remoteness [20]. See ESM for further details.

Selected comorbid conditions (diabetes, cardiac disease, hypertension, vascular disease, cerebrovascular disease and obesity) were flagged for each admission, based on the presence of relevant ICD 10 AM codes (ESM Table $\mathrm{S} 2$ ). Once present, a condition was carried forward to subsequent episodes.

Other exposure variables included the dialysis model of care $(\mathrm{DxMoC})$ and dialysis attendance, which was both an exposure and outcome variable.

The dialysis model of care was determined through a combination of admission ward, treatment option based on ICD 10 AM diagnosis or procedure codes (ESM Table S3) and ANZDATA treatment coding to determine the 'best fit'. As the intent of our study was to explore the relationship between dialysis model and subsequent health care use, determining the dominant model was critical and needed to accommodate the frequent movement of individuals between both facilities and modalities. This ws particularly important for the remote community-controlled model (DxMoC4) which operated primarily as a staffed reverse respite model, enabling individuals originally from remote areas to have short periods (2-6weeks) of dialysis in their own community. Therefore, the dominant DxMoC was based on the model in which the majority of attendances had occurred in the current and preceding two-week period, rolling forward a week at a time. This process, while eliminating extremely frequent movements between models, still enabled the respite model (DxMoC4) of 2-6weeks to be captured in the data.

Exposure to a model was then based on the time (in weeks) spent in the dominant DxMoC on an annual basis. Exposure time was censored at death or permanent loss to follow-up, defined as absence of any activity (dialysis or hospital admissions) without re-appearance before study end.

Dialysis attendance rates were calculated from the total attendances (based on $3 \mathrm{x}$ week or a proportion thereof) while in a DxMoC and divided by the exposure time (in weeks) for that model. This was then multiplied by fiftytwo (52 weeks) to calculate an attendance rate per year per DxMoC. The calculation excluded time as an inpatient, time as a transplant patient and time interstate, as identified from the patient travel data. 
Dialysis attendance was also categorised using three levels:

- 'High' (greater than or equal to 144 treatments a year from 156 prescribed treatments, i.e. missing one or fewer/month)

- 'Medium' (between 132 and 143 treatments, i.e. missing between one and two/month) and

- 'Low' (less than or equal to 131 treatments a year, i.e. missing more than two/month).

Definition of outcome and exposure variables are available at ESM Table S4.

Hospital admissions, Emergency Department (ED) presentations and days in hospital were summed by year and by model to provide respective utilisation estimates per person per model of care. We did not differentiate hospital admissions and ED presentations that occurred in the urban tertiary hospitals from the rural hospitals. We also examined diagnosis codes for each admission episode and isolated those likely to be associated with low dialysis attendance (based on ICD-10AM codes for Fluid Overload (E87.7) and Hyperkalaemia (E87.5)). These were summed by year and model.

A small number of incorrectly linked episodes $(n=5$ patients) were excluded. Nine $(n=9)$ haemodialysis patients had a gap of 12 months or more in the dataset with no haemodialysis attendance or hospital admission episodes, followed by a subsequent attendance. This gap was categorized as intermittent loss to follow up to cater for people who had extended periods interstate for medical care or were returning to dialysis after a failed transplant. Intermittent loss to follow up periods were not included in the model exposure calculation. Duplicate attendances (primarily from the overlap of dialysis attendance in the hospital data set and the additional, manually compiled data set for DxMoC4/5) were removed.

\section{Statistical analysis}

All analyses were conducted using Stata15.1@ (StataCorp., College Station; Texas USA). Simple linear regression analyses examined the relationship between annual dialysis attendance and gender, relocation, region, remoteness of residence pre-KRT start, age at admission, time on dialysis and DxMoC. Using both forward variable selection and backward variable elimination multivariate linear regression analyses, we tested the variables' tolerance for inclusion or exclusion in the model based on the $\mathrm{R}$ squared and confidence intervals. Sensitivity analysis included a range of interaction terms between DxMoC and other variables such as remoteness pre-KRT start, relocation, region, time on dialysis, admission age as well as select comorbidities.

Negative binomial regression was used to model the outcome variables of hospital admission rates and ED presentation rates and corresponding 95\% confidence intervals (CI) with all exposure variables mentioned above, as well as dialysis attendance as an exposure variable. Zero inflated poisson regression was used to model days in hospital, due to the frequency of zero counts.

\section{Results}

\section{Patient characteristics}

The final study population consisted of $n=896$ Aboriginal maintenance dialysis patients between the years 2008 to 2014. The cohort consisted of more females than males and most of the study population (82\%) were flagged as having relocated to start treatment. The age at start of KRT and median study observation time were similar for men and women (Table 2).

To assess potential differences in patient mix by DxMoC, we compared mean age and presence of comorbidities for each DxMoC. There was little difference across models for either age or comorbidities, although individuals attending In-centre models $(\mathrm{DxMoC} 0)$ were generally younger than those attending other models. The proportion of individuals with diabetes and vascular disease were also lower at In-centre DxMoC0 compared to the other models (Table 3). Full comorbidities by model of care are shown in ESM Table S5.

\section{DxMoC and Dialysis attendance}

Gender, remote residence pre-KRT start, patient age (less than 59 years), living in Central Australia (CA) and time on dialysis of less than four years were individually associated with lower yearly dialysis attendance $(<132$ treatments) as was dialysing at the In-centre model compared to all other models (mean 129-147 vs 97, $\mathrm{P}<0.001$ ). Full results are presented in ESM Table S6. When adjusted for gender, region, residence pre-KRT, admission age, time on dialysis and $\mathrm{DxMoC}$, these associations persisted (Fig. 3).

\section{Dialysis attendance and hospital admissions}

When dialysis attendance was stratified into a categorical variable, we found $43 \%$ of all attendances fell into the 'High' ( $>=144$ treatments), 15\% into the 'Medium' (132$143)$ and $42 \%$ into the 'Low' ( $<=131$ treatments) attendance categories although there was substantial variation by $\mathrm{DxMoC}$. The proportion of attendances that fell into the 'High' category were highest in DxMoC2,3 and 4 while In-centre DxMoC0 had the highest proportion of 'Low' attendances followed by Urban DxMoC1. 
Table 2 Patient characteristics at study entry of NT Aboriginal KRT patients, 2008-2014

\begin{tabular}{|c|c|c|c|}
\hline Characteristics & & Female & Male \\
\hline Full study cohort n (\%) & $n=896$ & $528(59)$ & $368(41)$ \\
\hline \multicolumn{4}{|l|}{ Region } \\
\hline Top End n (\%) & $434(48)$ & $259(49)$ & $175(48)$ \\
\hline Central Australia n (\%) & $462(52)$ & $269(51)$ & $193(52)$ \\
\hline \multicolumn{4}{|l|}{ Median time on *KRT (in years) } \\
\hline At study start (IQR) $(n=376)$ & $2.7(1.2-6.0)$ & $2.5(1.3-5.5)$ & $3.0(1.2-6.6)$ \\
\hline Study period (IQR) $(n=896)$ & $4.9(2.8-6.9)$ & $4.9(2.8-6.8)$ & $4.9(2.7-6.9)$ \\
\hline Mean age in years at KRT start (SD) & $47.6(11.3)$ & $48.2(11.5)$ & $46.7(11.0)$ \\
\hline Relocated n (\%) & $733(82)$ & $429(81)$ & $304(83)$ \\
\hline Residence pre-KRT (very remote) n (\%) & $697(78)$ & 409 (77) & $288(78)$ \\
\hline \multicolumn{4}{|l|}{ Comorbid conditions } \\
\hline Diabetes n (\%) & $731(82)$ & $441(84)$ & $290(79)$ \\
\hline Cardiac n (\%) & $451(50)$ & $259(49)$ & $192(52)$ \\
\hline Hypertension n (\%) & $830(93)$ & $489(93)$ & 341 (93) \\
\hline Cerebrovascular n (\%) & $46(5)$ & $25(5)$ & $21(6)$ \\
\hline Vascular n (\%) & $309(34)$ & $186(35)$ & $123(33)$ \\
\hline Obesity n (\%) & $109(12)$ & $59(11)$ & $50(14)$ \\
\hline
\end{tabular}

${ }^{*}$ KRT = Kidney Replacement Therapy

Table 3 Characteristics of Aboriginal patients at time of admission to DxMoC, 2008-2014

\begin{tabular}{|c|c|c|c|c|c|c|c|}
\hline & $\begin{array}{l}\text { In-centre } \\
\text { DxMoco }\end{array}$ & $\begin{array}{l}\text { Urban } \\
\text { DxMoC1 }\end{array}$ & $\begin{array}{c}\text { Rural } \\
\text { DxMoC2 }\end{array}$ & $\begin{array}{l}\text { Remote } \\
\text { DxMoC3 }\end{array}$ & $\begin{array}{l}\text { RemoteCC* } \\
\text { DxMoC4 }\end{array}$ & $\begin{array}{l}\text { SC HD* } \\
\text { DxMoC5 }\end{array}$ & $\begin{array}{c}\text { SC PD* } \\
\text { DxMoC6 }\end{array}$ \\
\hline Patients experiencing $\mathrm{DxMoC}=\mathrm{n}$ & 616 & 776 & 198 & 41 & 131 & 109 & 117 \\
\hline Mean age in years (SD) & $49.6(10.9)$ & $52.2(10.9)$ & $52.3(10.5)$ & $54.7(7.7)$ & $53.9(10.9)$ & $51.1(10.0)$ & $49.9(11.8)$ \\
\hline \multicolumn{8}{|l|}{ Comorbidities } \\
\hline Diabetes \% (95\% Cl) & $80(77-82)$ & $86(85-87)$ & $89(86-91)$ & $83(76-89)$ & $92(88-95)$ & $87(82-91)$ & $74(69-79)$ \\
\hline Cardiac \% $(95 \% \mathrm{Cl})$ & $57(54-60)$ & $61(59-63)$ & $69(65-72)$ & $61(52-69)$ & $55(54-61)$ & $76(70-81)$ & $60(54-65)$ \\
\hline Vascular \% $(95 \% \mathrm{Cl})$ & $45(42-48)$ & $59(57-60)$ & $58(54-62)$ & $55(47-64)$ & $61(55-66)$ & $61(55-68)$ & $60(54-65)$ \\
\hline Obesity \% (95\% Cl) & $13(11-16)$ & $18(17-20)$ & $12(10-15)$ & $10(6-17)$ & $19(14-26)$ & $16(12-21)$ & $11(8-15)$ \\
\hline
\end{tabular}

${ }^{*}$ CC:Community-controlled; SC HD: self-care haemodialysis; SC PD: self-care peritoneal dialysis

On examination of the reasons for hospitalisations, (based on Australian Refined Diagnosis Related Groups [21]), we noted that while the majority (95\%) of admissions contained a renal related diagnosis code, $25 \%$ of all admissions included one or both ICD-10 AM code for fluid overload (E87.7) or hyperkalaemia (E87.5) and a very high proportion of patients $(78 \%$ or $n=695)$ had at least one admission with the above code. While admissions for fluid overload/hyperkalaemia can be unrelated to missing haemodialysis treatments, these coded admissions were higher for patients who had 'Low' dialysis attendance (72\%) compared to 'High' dialysis attendance (13\%). The proportion of admissions by DxMoC containing these codes (which we categorised as 'Missed Dialysis') were highest in the urban models (Table 4). Mean (with 95\% CI) and median (with range) hospital admissions by an individual's exposure time to a specific DxMoC are also presented in Table 4.

Multivariate negative binomial regression showed a significant increased rate of both hospitalisations (IRR=2.10; 95\% CI:1.96-2.28) and ED presentations 


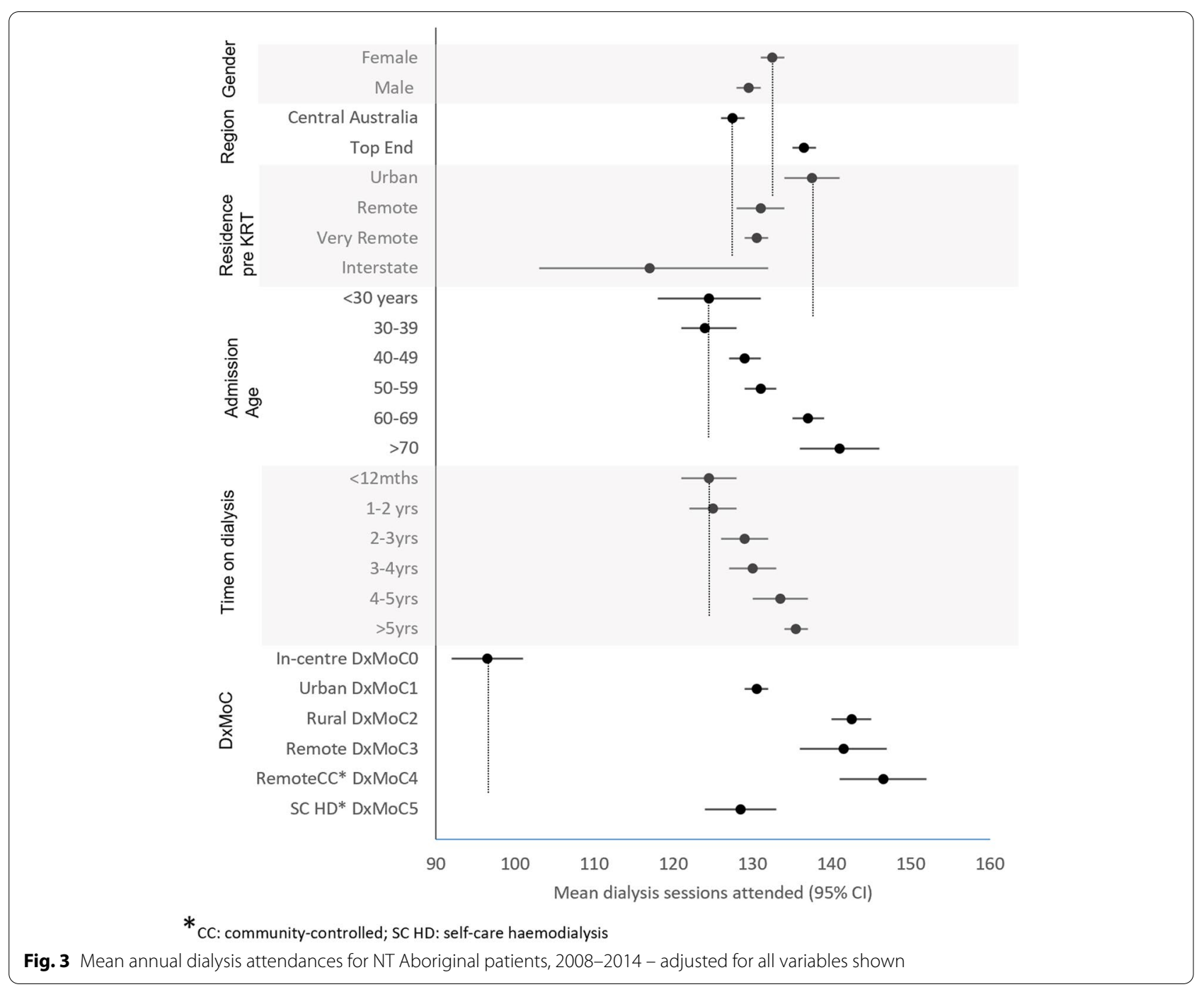

Table 4 Dialysis attendance and hospital admissions for NT Aboriginal patients by DxMoC, 2008-2014

\begin{tabular}{|c|c|c|c|c|c|c|c|}
\hline Dialysis attendance and hospital admissions & $\begin{array}{l}\text { In-centre } \\
\text { DxMoc0 }\end{array}$ & $\begin{array}{c}\text { Urban } \\
\text { DxMoC1 }\end{array}$ & $\begin{array}{c}\text { Rural } \\
\text { DxMoC2 } \\
\end{array}$ & $\begin{array}{l}\text { Remote } \\
\text { DxMoC3 }\end{array}$ & $\begin{array}{c}\text { RemoteCC* } \\
\text { DxMoC4 }\end{array}$ & $\begin{array}{c}\text { SC HD* } \\
\text { DxMoC5 }\end{array}$ & $\begin{array}{c}\text { SC PD* } \\
\text { DxMoC6 }\end{array}$ \\
\hline \multicolumn{8}{|l|}{ Dialysis attendance by category $\%$} \\
\hline High >=144 & 21 & 40 & 57 & 76 & 56 & 47 & \\
\hline Medium 132-143 & 10 & 17 & 16 & 10 & 16 & 20 & \\
\hline Low $<=131$ & 69 & 43 & 27 & 14 & 28 & 33 & \\
\hline Missed Dialysis ${ }^{a} \mathrm{n}$ (\% DxMoC admissions) & $752(24)$ & $4349(32)$ & $287(12)$ & $29(8)$ & $81(18)$ & $58(9)$ & $91(6)$ \\
\hline \multicolumn{8}{|l|}{ Hospital admissions/patient/DxMoC } \\
\hline Median (range) & $1(0-24)$ & $2(0-42)$ & $1(0-27)$ & $1(0-7)$ & $0(0-12)$ & $1(0-8)$ & $1(0-21)$ \\
\hline \multicolumn{8}{|c|}{ Mean $(95 \% \mathrm{Cl}) 1.6(1.5-1.7) 2.9(2.8-3.1) 2.3(2.1-2.6) 1.0(0.8-1.3) 0.9(0.7-1.1) 1.1$ (0.9-1.2) 1.9 (1.6-2.1 } \\
\hline
\end{tabular}


(IRR=3.29; 95\% CI :2.86-3.80) associated with 'Low' dialysis attendance ( $<132$ treatments) compared to high dialysis attendance ( $>143$ treatments). Results are presented in ESM Table S7.

\section{DxMoC, hospital admissions and ED presentations}

We modelled overnight hospital admissions and ED presentations separately, as an outcome with a range of exposure variables (without dialysis attendance and with self-care PD DxMoC6) using univariate and multivariate negative binomial regression. Unadjusted, region, remoteness of residence pre-KRT start, age less than 40 years, time on dialysis of less than four years, comorbidities of diabetes, cardiac disease, vascular disease and obesity as well as dialysing at In-centre DxMoC0 were all associated with an increased rate of overnight hospital admissions. These associations persisted in the multivariate analysis except for obesity, which was no longer significant, while time on dialysis greater than 12 months, was now associated with reduced rates of hospitalisation. We used Urban DxMoC1 as the reference due to the higher patient numbers attending this model (Table 5).

Results for ED presentations are presented in Supplementary materials (ESM Table S8) and found generally similar results with respect to significantly lower rates of ED presentations for rural and remote dialysis models of care. Results for days in hospital are also presented in Supplementary materials (ESM Table S9).

\section{Discussion}

Renal patients have high all-cause mortality and hospitalisation rates [22], usually related to cardiovascular events and infection. However we also showed a relationship between dialysis attendance, hospitalisations and DxMoC.

Our analysis of linked hospital clinical and administrative data for Aboriginal dialysis patients found a clear relationship between the location/type of treatment and dialysis attendance, and in turn these were both strongly associated with health service utilisation, particularly overnight hospital admissions. Our analysis did not include non-Aboriginal people as very few in the NT are from remote areas and the impact of relocation on health service utilisation and outcomes is not relevant to their experience of dialysis.

The relationship between missed or shortened dialysis treatments and hospitalisations has been explored previously. In one US study, missed and shortened treatments for a very small proportion of the study population (2.4\%) were associated with progressively more hospitalisations although the relative risks (RR) were not provided [16]. A comparable Australian study described a significant and consistent increase in hospitalisations associated with missing one treatment a week (i.e. four per month) with an IRR $=1.90$ in the first year [23]. Our analysis found that missing two or more treatments per month was associated with a two-fold increase in the rate of hospital admission and more than threefold increase in the rate of ED presentations. Additionally, the proportion of patients who missed one or more dialysis attendance a month was relatively high at $42 \%$ or greater, compared to the United States (US) where $7.9 \%$ (in the Dialysis Outcomes and Practice Patterns Study) was considered high [16].

The relationship between the location of dialysis treatment, missed dialysis treatments and hospitalisation rates has not been extensively explored to date. Our study included an analysis of geographical factors relating to original residence and location of service. We found relocation and remoteness of residence pre-KRT start, as well as younger age, were associated with lower dialysis attendance, a finding that is not uncommon in Northern Australia [13].

'Region' was also a strong predictor of health service utilisation, with Central Australian patients 30\% more likely to be admitted overnight and with a $70 \%$ higher risk of an ED presentation compared to patients in the Top End. These differences persisted after adjusting for age, time on dialysis, comorbidities and DxMoC.

However we found higher dialysis attendance and lower hospital admissions and ED presentations for Aboriginal patients receiving care in rural, remote and self-care haemodialysis models. While rural and remote services are usually reserved for clinically stable patients, we found no significant difference in the characteristics (age or comorbidities) of patients attending urban versus rural and remote models of care, indicating that age and the presence of comorbid conditions do not completely account for the higher rates of hospital admissions seen at In-centre DxMoC0 and Urban DxMoC1. Perhaps it also suggests that the low dialysis attendance associated with relocation and care in urban models can potentially be ameliorated by access to rural and remote models.

Patients being treated with self-care haemodialysis (DxMoC5) had lower dialysis attendance patterns on average but not increased health service utilisation. Although we made additional efforts to capture all relevant data, we believe the lower dialysis attendance for this group may have resulted from missing data.

To date, evidence on how models of care influence the quality of care and patient outcomes has been variable. Some evidence suggests that hospital presentations, along with an increased risk of morbidity and mortality, are associated with specific models of care, particularly remote based facilities where there is low access to nephrologists and significant distance to a tertiary service [24, 
Table 5 Hospital admission incident rate ratios (IRR) for NT Aboriginal patients, 2008-2014

\begin{tabular}{|c|c|c|c|c|c|}
\hline \multirow{2}{*}{\multicolumn{2}{|c|}{$\begin{array}{l}\text { Negative binomial regression } \\
\text { adjusted for included variables }\end{array}$}} & \multicolumn{4}{|c|}{ Hospital Admission Incidence Rate Ratio (IRR) } \\
\hline & & \multicolumn{2}{|c|}{ Univariate } & \multicolumn{2}{|c|}{ Multivariate } \\
\hline & & $\operatorname{IRR}(95 \% \mathrm{Cl})$ & $\underline{P \text { value }}$ & $\operatorname{IRR}(95 \% \mathrm{Cl})$ & $\underline{\text { P Value }}$ \\
\hline Gender & Male (vs Females) & $1.01(0.96-1.07)$ & 0.631 & $1.02(0.95-1.08)$ & 0.649 \\
\hline Region & TE (vs CA)* & $0.65(0.62-0.69)$ & $<0.001$ & $0.71(0.66-0.76)$ & $<0.001$ \\
\hline Residence pre- & Urban & 1 & Reference & 1 & Reference \\
\hline \multirow[t]{3}{*}{ KRT } & Remote & $2.11(1.86-2.38)$ & $<0.001$ & $1.44(1.24-1.69)$ & $<0.001$ \\
\hline & Very Remote & $1.65(1.48-1.83)$ & $<0.001$ & $1.39(1.22-1.57)$ & $<0.001$ \\
\hline & Interstate & $3.64(2.56-5.17)$ & $<0.001$ & $2.11(1.43-3.12)$ & $<0.001$ \\
\hline \multirow[t]{6}{*}{ Admission age } & $<30 y r s$ & 1 & Reference & 1 & Reference \\
\hline & 30-39yrs & $1.37(1.16-1.61)$ & $<0.001$ & $1.17(0.97-1.42)$ & 0.098 \\
\hline & $40-49 y r s$ & $0.86(0.74-1.00)$ & 0.053 & $0.78(0.65-0.93)$ & 0.006 \\
\hline & $50-59 y r s$ & $0.80(0.69-0.93)$ & 0.004 & $0.71(0.59-0.84)$ & $<0.001$ \\
\hline & $60-69 y r s$ & $0.78(0.67-0.92)$ & 0.002 & $0.73(0.61-0.88)$ & 0.001 \\
\hline & $>70 y r s$ & $0.63(0.51-0.78)$ & $<0.001$ & $0.64(0.50-0.81)$ & $<0.001$ \\
\hline \multirow[t]{6}{*}{ Time on dialysis } & $<12$ mths & 1 & Reference & 1 & Reference \\
\hline & $1-2$ yrs & 1.25 (1.14-1.37) & $<0.001$ & $0.70(0.63-0.79)$ & $<0.001$ \\
\hline & $2-3 y r s$ & 1.16 (1.05-1.29) & 0.005 & $0.63(0.58-0.71)$ & $<0.001$ \\
\hline & $3-4 y r s$ & $1.18(1.06-1.31)$ & 0.002 & $0.65(0.57-0.73)$ & $<0.001$ \\
\hline & $4-5 y r s$ & $1.03(0.91-1.15)$ & 0.652 & $0.54(0.47-0.62)$ & $<0.001$ \\
\hline & $>5 y r s$ & $1.05(0.97-1.14)$ & 0.194 & $0.56(0.50-0.62)$ & $<0.001$ \\
\hline \multirow{4}{*}{$\begin{array}{l}\text { Comorbid } \\
\text { conditions }\end{array}$} & Diabetes & $1.19(1.10-1.28)$ & $<0.001$ & $1.22(1.11-1.35)$ & $<0.001$ \\
\hline & Cardiac & $1.32(1.25-1.40)$ & $<0.001$ & $1.32(1.23-1.42)$ & $<0.001$ \\
\hline & Vascular & $1.41(1.33-1.50)$ & $<0.001$ & $1.39(1.30-1.50)$ & $<0.001$ \\
\hline & Obesity & $1.21(1.13-1.31)$ & $<0.001$ & $1.04(0.96-1.13)$ & 0.353 \\
\hline \multirow[t]{7}{*}{ DxMoC } & In-centre DxMoCo & $1.21(1.22-1.30)$ & $<0.001$ & $2.19(1.98-2.42)$ & $<0.001$ \\
\hline & Urban DxMoC1 & 1 & Reference & 1 & Reference \\
\hline & Rural DxMoC2 & $0.94(0.86-1.02)$ & 0.152 & $0.92(0.83-1.01)$ & 0.101 \\
\hline & Remote DxMoC3 & $0.48(0.38-0.55)$ & $<0.001$ & $0.56(0.45-0.71)$ & $<0.001$ \\
\hline & RemoteCC* DxMoC4 & $0.94(0.83-1.07)$ & 0.358 & $0.62(0.50-0.72)$ & $<0.001$ \\
\hline & SC HD* DxMoC5 & $0.50(0.43-0.57)$ & $<0.001$ & $0.54(0.46-0.64)$ & $<0.001$ \\
\hline & SC PD* DxMoC6 & $0.74(0.66-0.84)$ & $<0.001$ & $0.82(0.71-0.94)$ & 0.004 \\
\hline
\end{tabular}

*TE: Top End; CA: Central Australia; CC:Community-controlled; SC HD: self-care haemodialysis; SC PD: self-care peritoneal dialysis

25]. However, in many regions/jurisdictions, movement between satellite units and hospitals is common, with an increased risk of hospitalisation associated with certain satellite facilities, not necessarily remote facilities [26] and not necessarily related to missed treatments [27].
Given the vastness of the NT, the number of remote communities and the limited services in rural and remote areas, there are bound to be differing degrees of relocation. People receiving services in rural areas may still have relocated from a very remote area. They may therefore face the same issues relating to the cost and time of 
travel to attend community activities, leading to missed treatments. The examination of attendance patterns and health service use by the degree of dislocation is an area for future analysis.

Our study found reduced rates of health service utilisation associated with dialysing in rural and remote locations. There is an argument that for remote living individuals, treatment within community improves outcomes $[7,28]$. Patients maintain that these models, which provide care closer to home, are more supportive of the health and wellbeing of individuals, which in turn facilitates more active engagement with treatment [12, 29-32] leading to lower health service utilisation.

\section{Limitations}

We are aware that validation studies for the use of ICD-10 coding to identify kidney disease (usually acute kidney injury or chronic kidney disease) have indicated poor congruence between diagnosable conditions and documentation [33,34] and acknowledge that the coding of peritoneal dialysis (PD) and transplantation is likely to be of similarly reduced quality. The linkage with the ANZDATA set, however, has addressed this limitation. The coding of haemodialysis in the NT is relatively robust as it is the primary reason for admission and required for activity-based funding. Additionally, recent local validation studies in the NT improved the quality of this coding [35]. We also took extra steps to assess the validity of the data and source additional data sets where there was prior knowledge of data gaps. However we did not interpolate data where data gaps remained, as we could not be certain activity had occurred. In retaining the participant, such data gaps were designated intermittent LTFU.

Although we found few differences in age or presence of comorbidities across the models, we recognise that clinician and patient self-selection may limit access to remote models for more frail patients, particularly where there is limited facility capacity and waiting lists exist. We also recognise that low dialysis attendance is but one cause of poorer health and higher hospitalisations; renal patients are known to experience high rates of hospitalisations due to other factors, usually arising from complications associated with cardiac disease and sepsis [22, 36]. Nevertheless, our results suggest a statistically and clinically significant impact of dialysis attendance on hospitalisations and ED presentations.

\section{Conclusion}

There is a significant gap in knowledge concerning the relationship between the provision and uptake of dialysis services in urban, rural and remote areas and subsequent broader health service utilisation. In the NT, Aboriginal people from remote areas have a very heavy burden of kidney failure requiring kidney replacement therapy.

The situation necessitates a re-examination of service provision and the identification of ways to deliver accessible, equitable and high-quality services that meet patients' health, social and cultural needs.

This study highlights the differences in the attendance patterns and health service use associated with dialysis treatment in different locations and particularly for Aboriginal people originally from rural and remote areas. More broadly, this analysis also illustrates the impact of policy decisions on health service utilisation. Decisions to limit the variety of models of dialysis care available to patients may be understandable, particularly in remote areas, given constraints related to volume/demand, infrastructure capacity and costs of service provision. However, our analysis suggests that policy decisions that do not consider and appreciate the full range of intended and unintended consequences, including downstream health service utilisation, of various service models, may lead to suboptimal decisions about allocation of scarce resources.

\section{Supplementary Information}

The online version contains supplementary material available at https://doi. org/10.1186/s12913-022-07628-9.

\section{Additional file 1.}

\section{Acknowledgements}

The author would like to thank Dr. Jeannie Devitt for her advice, guidance and assistance with manuscript preparation.

\section{Authors' contributions}

GG, PDL and AC designed the study, GG undertook the analysis with statistical advice and support from JC, KH, PDL, AMSA and FB. GG drafted the initial manuscript and $\mathrm{KH}, \mathrm{JC}$ and $\mathrm{AC}$ revised the structure. All authors critically reviewed the manuscript, contributed to the intellectual content and approved the final version of the manuscript.

\section{Funding}

The DxMoC study was funded through a National Health and Medical Research Council (NHMRC) grant (\#1092092). GG was supported with a National Health and Medical Research Council (NHMRC) Postgraduate Scholarship Grant (\#116826). JC was funded by an NHMRC Research Fellowship (\#1058244). All authors declared no competing interests.

\section{Availability of data and materials}

The data that supports the findings of this study were provided by the Northern Territory Department of Health, but restrictions apply to the availability of these data, which were conditions of the Ethics approval and so are not publicly available. With permission of the Northern Territory Department of Health, data are however available from the authors upon reasonable request. 


\section{Declarations}

\section{Ethics approval and consent to participate}

Ethics approval for the study was granted by the Joint Department of Health and Menzies School of Health Research Human Research Ethics Committee (HREC 2015-2334) and the Central Australian Health Research Ethics Committee (HREC 15-283). The study was reviewed and approved by their respective Aboriginal Ethics Sub-committees. The study was performed in accordance with the relevant guidelines and regulations of the relevant ethics committees and in accordance with the Declaration of Helsinki.

Informed consent was waived by the the Joint Department of Health and Menzies School of Health Research Human Research Ethics Committee (HREC 2015-2334) and the Central Australian Health Research Ethics Committee (HREC 15-283) and their respective Aboriginal sub-committees.

\section{Consent for publication}

Not applicable.

\section{Competing interests}

The authors report no conflicts of interest.

\section{Author details}

${ }^{1}$ Menzies School of Health Research, Charles Darwin University, Darwin 0810, Australia. ${ }^{2}$ Sydney School of Public Health, Faculty of Medicine and Health, The University of Sydney, Sydney, Australia. ${ }^{3}$ Menzies Centre for Health Policy and Economics, Faculty of Medicine and Health, The University of Sydney, Sydney, Australia. ${ }^{4}$ Primary Health Tasmania (Tasmania PHN), Hobart, Tasmania, Australia. ${ }^{5} \cup Q$ Poche Centre, University of Queensland, Brisbane, Queensland, Australia.

Received: 11 September 2021 Accepted: 9 February 2022 Published online: 24 February 2022

\section{References}

1. Garcia-Garcia G, Jha V. World kidney day steering C. chronic kidney disease in disadvantaged populations. Braz J Med Biol Res. 2015;48(5):377-81.

2. Brophy PD, Shoham DA, Charlton JR, Carmody JB, Reidy KJ, Harshman L, et al. Early-life course socioeconomic factors and chronic kidney disease. Adv Chronic Kidney Dis. 2015;22(1):16-23.

3. Cass A, Cunningham J, Snelling P, Wang Z, Hoy W. End-stage renal disease in indigenous Australians: a disease of disadvantage. Ethnicity Disease. 2002;12(3):373-8.

4. Australian Institute of Health and Welfare. Chronic kidney disease: regional variation in Australia. In: AlHW, editor. Canberra: Australian Government; 2013

5. ANZDATA Registry. 43rd report, chapter 2: prevalence of renal replacement therapy for end stage kidney disease. Adelaide, Australia: Australia and New Zealand Dialysis and Transplant Registry; 2020

6. Gorham G, Majoni S, Lawton P, Brown S, Dube B, Conlon T, et al. Interesting times: evolution of dialysis in Australia's Northern Territory (1980-2014). Renal Soc Australasia J. 2018;14(3):108-16.

7. Bernstein K, Zacharias J, Blanchard JF, Yu BN, Shaw SY. Model for equitable care and outcomes for remote full care hemodialysis units. Clin J Am Soc Nephrol. 2010;5(4):645-51.

8. Anderson K, Cunningham J, Devitt J, Preece C, Cass A. "looking back to my family": indigenous Australian patients' experience of hemodialysis. BMC Nephrol. 2012;13:114.

9. McGrath P. Exploring Aboriginal people's experience of relocation for treat ment during end-of-life care. Int J Palliat Nurs. 2006;12(3):102-8.

10. Lavoie JG, Kaufert J, Browne AJ, Mah S, O’Neil JD, Sinclair S, et al. Negotiating barriers, navigating the maze: first nation peoples' experience of medical relocation. Can Public Administration. 2015;58(2):295-314.

11. Wilson R, Krefting L, Sutcliffe P, Van Bussel L. Native Canadians relocating for renal dialysis. Psychosocial and cultural issues. Can Fam Physician. 1994;40:1934-41.

12. Diamant MJ, Harwood L, Movva S, Wilson B, Stitt L, Lindsay RM, et al. A comparison of quality of life and travel-related factors between in-center and satellite-based hemodialysis patients. Clin J Am Soc Nephrol. 2010;5(2):268-74

13. Smyth W, Hartig V, Hayes M, Manickam V. Patients' adherence to aspects of haemodialysis regimens in tropical North Queensland, Australia. J Ren Care. 2015:41(2):110-8

14. Anderson K, Devitt J, Cunningham J, Preece C, Jardine M, Cass A. If you can't comply with dialysis, how do you expect me to trust you with transplantation?, Australian nephrologists'views on indigenous Australians'non-compliance' and their suitability for kidney transplantation. Int J Equity Health. 2012;11:21.

15. Yan G, Norris KC, Greene T, Yu AJ, Ma JZ, Yu W, et al. Race/ethnicity, age, and risk of hospital admission and length of stay during the first year of maintenance hemodialysis. Clin J Am Soc Nephrol. 2014;9(8):1402-9.

16. Obialo Cl, Hunt WC, Bashir K, Zager PG. Relationship of missed and shortened hemodialysis treatments to hospitalization and mortality: observations from a US dialysis network. Clin Kidney J. 2012;5(4):315-9.

17. Australian Statistical Geography Standard (ASGS) [Internet]. ABS. 2016 [cited 22 August 2018]. Available from: Australian Statistical Geography Standard (ASGS) Volume 5 - Remoteness Structure (cat. no. 1270.0.55.005) publication.

18. Gorham G, Howard K, Togni S, Lawton P, Hughes J, Majoni SW, et al. Economic and quality of care evaluation of dialysis service models in remote Australia: protocol for a mixed methods study. BMC Health Serv Res. 2017;17(1):320.

19. Yuejen Z, John RC, Shu Qin L, Steven G, Ramakrishna C. Indigenous patient migration patterns after hospitalisation and the potential impacts on mortality estimates. Australas J Reg Stud. 2013;19(3):321.

20. Department of Health. Modified Monash Model: Australian Government; 2015 [updated 12 June 2018,. Available from: http://www.health.gov.au/ internet/main/publishing.nsf/content/modified-monash-model.

21. IPHA. The AR-DRG classification system [Available from: https://www.ihpa. gov.au/admitted-acute-care/ar-drg-classification-system.

22. Long B, Koyfman A, Lee CM. Emergency medicine evaluation and management of the end stage renal disease patient. Am J Emerg Med. 2017;35(12):1946-55.

23. Lawton PD, Cunningham J, Zhao Y, Jose MD. What's wrong with missing dialysis? Patterns and outcomes of dialysis attendance in the Northern Territory, 1995-2011. Nephrology. 2015;20(S2):58-9.

24. Thompson S, Gill J, Wang X, Padwal R, Pelletier R, Bello A, et al. Higher mortality among remote compared to rural or urban dwelling hemodialysis patients in the United States. Kidney Int. 2012;82(3):352-9.

25. Tonelli M, Manns B, Culleton B, Klarenbach S, Hemmelgarn B, Wiebe N, et al. Association between proximity to the attending nephrologist and mortality among patients receiving hemodialysis. Can Med J. 2007;177(9):1039-44.

26. Barthelemy A, Lobbedez T, Bechade C, Henri P, Batho JM, Cardineau E, et al. Hemodialysis in satellite dialysis units: incidence of patient fallback to the in-center dialysis unit. J Nephrol. 2015;28(4):485-93.

27. Mathew AT, Strippoli GF, Ruospo M, Fishbane S. Reducing hospital readmissions in patients with end-stage kidney disease. Kidney Int. 2015;88(6):1250-60

28. Zacharias J, Komenda P, Olson J, Bourne A, Franklin D, Bernstein K. Home hemodialysis in the remote Canadian north: treatment in Manitoba fly-in communities. Semin Dial. 2011:24(6):653-7.

29. Rix EF, Barclay L, Stirling J, Tong A, Wilson S. 'Beats the alternative but it messes up your life': aboriginal people's experience of haemodialysis in rural Australia. BMJ Open. 2014;4(9):e005945.

30. Marshall MR, van der Schrieck N, Lilley D, Supershad SK, Ng A, Walker RC, et al. Independent community house hemodialysis as a novel dialysis setting: an observational cohort study. Am J Kidney Dis. 2013;61(4):598-607.

31. Kneipp E, Murray R, Warr K, Fitzclarence C, Wearne M, Maguire G. Bring me home: renal dialysis in the Kimberley. Nephrology (Carlton, Vic). 2004;9 Suppl 4:S121-5.

32. Salvalaggio G, Kelly L, Minore B. Perspectives on health: experiences of first nations dialysis patients relocated from remote communities for treatment. Can J Rural Medicine. 2003;8(1):19-24.

33. Logan R, Davey P, De Souza N, Baird D, Guthrie B, Bell S. Assessing the accuracy of ICD-10 coding for measuring rates of and mortality from acute kidney injury and the impact of electronic alerts: an observational cohort study. CKJ Clin Kidney J. 2020;13(6):1083-90.

34. Kitsos A, Peterson GM, Jose MD, Khanam MA, Castelino RL, Radford JC. Variation in documenting diagnosable chronic kidney disease in general medica 
practice: implications for quality improvement and research. J Prim Care Community Health. 2019;10:2150132719833298.

35. You JQ., Lawton P., Zhao Y., Poppe S., Cameron N., Guthridge S. Renal replacement therapy demand study, Northern Territory, 2001 to 2022. Darwin: Department of Health; 2015.

36. You J, Hoy W, Zhao Y, Beaver C, Eagar K. End-stage renal disease in the Northern Territory: current and future treatment costs. Med J Aust. 2002;176(10):461-5

\section{Publisher's Note}

Springer Nature remains neutral with regard to jurisdictional claims in published maps and institutional affiliations.

- fast, convenient online submission

- thorough peer review by experienced researchers in your field

- rapid publication on acceptance

- support for research data, including large and complex data types

- gold Open Access which fosters wider collaboration and increased citations

- maximum visibility for your research: over $100 \mathrm{M}$ website views per year

At BMC, research is always in progress.

Learn more biomedcentral.com/submissions 